\title{
Ist die Praxis bevorzugter Anstellung moralisch zulässig? ${ }^{1}$
}

\section{Is Preferential Hiring Morally Permissible?}

\section{Christine Bratu, München}

Zusammenfassung: In diesem Aufsatz diskutiere ich die Frage, ob es moralisch zulässig ist, bei der Verteilung von Positionen in der akademischen Philosophie in Deutschland partiell vom Prinzip der Bestenauswahl abzuweichen, um Frauen bevorzugt anzustellen. Für Anhänger*innen der traditionellen Sichtweise, der zufolge diejenige Bewerber*in eine akademische Position bekommen sollte, die über das meiste Potential für diese Position verfügt, wäre dies eine ungerechtfertigte Form der Diskriminierung. Ich argumentiere, dass dieser Vorwurf nicht zutrifft, jedenfalls nicht, wenn wir davon ausgehen, dass die gegenwärtige Vergabepraxis von akademischen Positionen von Phänomenen wie implicit biases und stereotype threat verzerrt wird. Um diese These einzuholen, analysiere ich das meiner Ansicht nach beste Argument, das Anhänger*innen der traditionellen Sichtweise zur Verfügung steht, und zeige, dass dieses abgeschwächt werden muss. In seiner abgeschwächten Form ist dieses Argument aber nicht nur mit der bevorzugten Anstellung von Frauen vereinbar, diese setzt sogar die Ziele, die hinter der traditionellen Sichtweise stehen, besser um als die traditionelle Sichtweise selbst.

Schlagwörter: Chancengleichheit - Quote - Diskriminierung - implicit bias/stereotype threat - Meritokratie

1 Ich danke den Teilnehmer*innen der Sektion „Philosophie und Gender“ auf dem XXIII. Deutschen Kongress für Philosophie in Münster, des Workshops „Why are there so few women in philosophy and (why) does it matter" an der Universität Stockholm und des SWIP Germany Panels auf der Hypatia Conference Villanova, PA für ihr wertvolles Feedback ebenso wie zwei anonymen Gutachter*innen der ZfPP. Besonderer Dank gilt Anca Gheaus für zahlreiche hilfreiche Hinweise zu einer früheren Version dieses Aufsatzes, Andrea Klonschinksi für wertvolle Tipps für die Überarbeitung des Manuskripts nach seiner Annahme und Marie Bohlen für die sorgfältige Durchsicht der Fahnen. 
Abstract: In this paper I discuss whether the preferential hiring of women in German academic philosophy is morally permissible. The traditional view claims that we should always hire the candidate with the highest academic potential; according to this view, preferential hiring constitutes a form of illegitimate discrimination. I argue that this assessment is false, at least if we assume that academic philosophy is currently distorted by implicit biases and stereotype threat. To prove this, I analyze what I consider to be the best argument in favor of the traditional view and show that it needs to be amended. But in its amended version, this argument is compatible with preferential hiring. In fact, it turns out that preferential hiring serves the underlying assumptions of the traditional view better than the traditional view does itself.

Keywords: equality of opportunity - affirmative action - discrimination - implicit bias/stereotype threat - meritocracy

\section{Einleitung}

Ist es moralisch zulässig, bei der Verteilung von Positionen in der akademischen Philosophie in Deutschland eine bestimmte Form so genannter affirmative action $^{2} \mathrm{zu}$ praktizieren? Genauer gesagt: Ist es moralisch zulässig, bei der Verteilung von Promotions-, PostDoc- und Assistenzstellen sowie von Professuren im Fach Philosophie an deutschen Universitäten und Forschungsinstituten partiell vom Prinzip der Bestenauswahl abzuweichen, um Frauen bevorzugt anzustellen? Strategisch spricht einiges dagegen, sich an diese Frage zu wagen. Denn unabhängig davon, ob wir sie bejahen (wie ich es tun werde) oder verneinen, hängt es letztlich von juristischen Regelungen ab, ob bevorzugte Anstellung praktiziert werden kann oder nicht. Das Hochschulgesetz des Bundeslands, in dem ich selbst tätig bin, verbietet diese Praxis beispielsweise, wenn es die Durchsetzung der Gleichberechtigung von Frauen und Männern nur „unter Beachtung des Vorrangs von Eignung, Befähigung und fachlicher Leistung“ vorsieht (vgl. BayHSchG, Artikel 4, Absatz 1). Darüber hinaus mag es intellektuell unredlich erscheinen, wenn ich eine Praxis verteidige, von der ich selbst profitieren könnte. Denn oft gelingt es uns nicht besonders gut, Richter*innen in eigener Sache zu sein. Und schließlich legt obige Frage eine Annahme nahe, die Feminist*innen und

2 Unter affirmative action wird gemeinhin eine Reihe unterschiedlicher Maßnahmen zur Besserstellung unterrepräsentierter sozialer Gruppen verstanden wie etwa die gezielte Förderung oder Anwerbung deren Mitglieder, vor allem aber auch deren bevorzugte Anstellung (vgl. Fullinwider 2018). Im Folgenden wird es ausschließlich um Letzteres gehen. 
vernünftige Menschen im Allgemeinen seit jeher mit Nachdruck ablehnen, nämlich dass Frauen intellektuell weniger leistungsfähig sind als Männer.

Mir scheint es dennoch lohnend, die Praxis bevorzugter Anstellung (im Folgenden PBA) zu verteidigen. Als Philosoph*innen müssen wir uns nämlich nicht auf den juristischen Status quo festlegen, sondern dürfen darüber nachdenken, wie unsere Gesetze beschaffen sein sollten. Und auch wenn meine nachfolgenden Überlegungen zugegebenermaßen eine Position rechtfertigen, die Personen wie mich bevorteilen könnte, haben sie zudem den intellektuellen Mehrwert aufzuzeigen, welche normativen Annahmen man teilen muss, um PBA zu verteidigen. Dadurch nutzen sie auch denjenigen, die diese Art der affirmative action für moralisch unzulässig halten, da sie deutlich machen, an welcher Stelle man sie am besten kritisiert. Die zuletzt genannte Sorge schließlich ist in meinen Augen einfach ein non sequitur. Denn die These, dass es moralisch zulässig ist, für die bevorzugte Anstellung von Frauen partiell vom Prinzip der Bestenauswahl abzugehen, impliziert für sich genommen noch nichts über die Befähigung von Frauen. Für Erstes zu argumentieren bedeutet nicht, dass man zu Zweitem eine bestimmte Position vertritt.

Der Grund, warum man sich für die bevorzugte Anstellung von Frauen in der akademischen Philosophie interessieren sollte (unabhängig davon, ob man potentielle Nutznießerin ist), ist die andauernde Unterrepräsentation von Frauen in diesem Bereich. Zugegebenermaßen ist dieses Phänomen für den englischsprachigen Raum besser dokumentiert als für Deutschland (vgl. Antony 2012; Mackenzie und Townley 2013 sowie Baron et al. 2015). Doch nach vorläufigen Erhebungen waren im Jahr 2013 nur zirka 17 Prozent aller Philosophieprofessuren in Deutschland von Frauen besetzt (vgl. Mikkola 2013, 4) bzw. 22 Prozent im Jahre 2016 (vgl. Klonschinski 2018) - und das, obwohl das Geschlechterverhältnis unter den Studierenden der Philosophie ausgeglichen ist (vgl. Klonschinski 2018). Spätestens mit der Promotion klafft die Schere zwischen den Geschlechtern allerdings auseinander, so dass bspw. der Anteil der von Frauen abgeschlossenen Promotionen im Jahre 2016 lediglich bei 32 Prozent liegt. Damit ist die Repräsentation von Frauen in der akademischen Philosophie weit schlechter als in den übrigen Geisteswissenschaften, zu denen die Philosophie häufig gezählt wird, und eher mit der in der Mathematik oder im Wirtschaftsingenieurswesen vergleichbar (vgl. Klonschinski 2018). Wie gesagt: Diese Zahlen gehen auf vorläufige Erhebungen zurück und umfassendere Untersuchungen wären sehr wünschenswert. Aber vielleicht zeigt die Tatsache, dass weder die Deutsche 
Gesellschaft für Philosophie noch die Gesellschaft für analytische Philosophie bisher versucht haben, systematisch Datenerhebung und Ursachenforschung zu betreiben und dadurch den Verdacht, dass die akademische Philosophie ein Problem mit Geschlechtergerechtigkeit hat, zu entkräften, bereits an sich, welche Priorität dieser Frage eingeräumt wird.

Ob affirmative action im Allgemeinen und PBA im Besonderen moralisch zulässige Maßnahmen sind, wird seit den 7oer Jahren heftig diskutiert, als diese Praktiken in den USA umfassend Verbreitung fanden (die klassischen Texte der Debatte hat Beate Rössler in deutscher Übersetzung zusammengetragen in Rössler 1993). Dass affirmative action mittlerweile auch in den USA legislativ unter Beschuss gerät, spiegelt die argumentative Lage in der philosophischen Debatte wider. Denn vielen erscheint insbesondere PBA eine Form unzulässiger Diskriminierung zu sein (vgl. Newton 1973; Pojman 1998; Gräfrath 1999). Gegen diese Auffassung will ich mich mit dem vorliegenden Text wenden. Hierfür stelle ich zuerst die traditionelle Sichtweise dar, der zufolge diejenige Bewerber*in eine akademische Position bekommen sollte, die über das größte akademische Potential für diese Position verfügt, sowie zwei Versionen von PBA, um dann den grundlegenden Dissens dieser Positionen herauszuarbeiten (2). Im Anschluss präsentiere ich zwei mögliche Argumente für die traditionelle Sichtweise (3). Diese Argumente werden zwar nicht explizit von Anhänger*innen dieser Position vorgebracht, da sich diese oft damit begnügen, Argumente gegen affirmative action anzuführen (vgl. Gräfrath 1999; ein Anhänger der traditionellen Sichtweise, der diese auch positiv verteidigt, ist dagegen Pojman 1998); aber sie rekonstruieren das, was Anhänger*innen der traditionellen Sichtweise wahrscheinlich im Sinn haben. Daraufhin lege ich in (4) zum einen dar, dass das beste Argument für die traditionelle Sichtweise dafür spricht, diese teilweise zu modifizieren. Zum anderen zeige ich, dass die traditionelle Sichtweise in ihrer modifizierten Version nicht nur mit PBA vereinbar ist, sondern dass PBA die Ziele der traditionellen Sichtweise sogar besser umsetzt als diese selbst - zumindest unter den aktuell in der akademischen Philosophie in Deutschland herrschenden Umständen. In (5) diskutiere und widerlege ich einen möglichen Einwand gegen meine Überlegungen und in (6) schließe ich, indem ich aufzeige, für welche weiteren Gegenstandsbereiche sich meine Überlegungen nutzbar machen lassen.

Vorweg muss ich klären, wen ich im Weiteren mit dem Begriff „Frau“ bezeichnet wissen will, d.h., für wessen bevorzugte Anstellung ich eigentlich plädiere. In Abweichung vom aktuell verbreiteten Sprachgebrauch verstehe 
ich unter einer Frau eine Person, die sich weiblich präsentiert und also über einen andauernden Zeitraum hinweg als Frau auftritt, unabhängig davon, welches biologische Geschlecht dieser Person bei ihrer Geburt zugewiesen wurde. Ein solches transinklusives Verständnis scheint mir richtig und zudem politisch geboten. ${ }^{3}$

\section{Die traditionelle Sichtweise und die Praxis bevorzugter Anstellung}

Grundsätzlich geht es sowohl den Befürworter*innen als auch den Kritiker*innen von PBA um die Frage, nach welchem Prinzip wir akademische Positionen verteilen dürfen, wenn wir der Gerechtigkeit Genüge tun wollen. Den Kritiker*innen zufolge sollte bei der Verteilung akademischer Positionen zum einen ausschließlich das akademische Potential der Bewerber*innen berücksichtigt werden; zum anderen sollte das akademische Potential der Bewerber*innen maximiert werden, d.h., es sollte diejenige Bewerber*in ausgewählt werden, die am meisten akademisches Potential hat. Dabei ist mit dem akademischen Potential der Bewerber*innen Folgendes gemeint: Eine Bewerber*in A hat akademisches Potential für eine akademische Position P genau dann, wenn/weil A die Fähigkeit(en) hat, P erfolgreich auszufüllen, wobei es von der Beschaffenheit von P abhängt, welche Fähigkeit(en) A haben muss, um P erfolgreich auszufüllen (für eine ähnliche Auffassung von Potential vgl. Hull 2015, 114). Um etwa eine Professur im Fach Philosophie erfolgreich auszufüllen, muss A (u.a.) die Fähigkeiten haben, interessante philosophische Texte zu schreiben, philosophische Argumente klar (und im besten Fall mitreißend) mündlich und schriftlich darzulegen und in Diskussionen konstruktiv Feedback zu geben. Verschiedene Bewerber*innen können die für eine akademische Position ausschlaggebenden Fähigkeiten in unterschiedlichem Maße vorweisen, so dass sie mehr oder weniger akademi-

3 In diesem Artikel behandle ich also nicht die Fragen, ob die bevorzugte Anstellung von Personen, die non-binary präsentieren, oder von people of colour moralisch zulässig ist. An sich sind diese Fragen genauso wichtig wie die, der ich mich widme. Ich beschränke mich dennoch auf Frauen, da die genannten Gruppen in der deutschen akademischen Philosophie nach wie vor sozial so „unsichtbar“ sind, dass zu ihrer Repräsentation keine Zahlen existieren. Sollte sich herausstellen, dass Mitglieder dieser sozialen Gruppen in der deutschen akademischen Philosophie unterrepräsentiert sind (was mir intuitiv sehr plausibel erscheint), gilt das Argument, das ich hier vorbringe, mutatis mutandis auch für sie. 
sches Potential für die fragliche Position haben. Allerdings gehen in der akademischen Philosophie die Auffassungen dazu, was einen interessanten Text ausmacht oder wann ein Argument klar dargelegt wurde, häufig auseinander (vgl. Hutchison 2013, 118f., sowie Gheaus 2015, 170). Daher wird meist auf andere, leichter zu ermittelnde Merkmale als Indikatoren für akademisches Potential zurückgegriffen, wie etwa an welchen Institutionen eine Bewerber*in ihre philosophische Ausbildung erhalten hat, wie viele Aufsätze sie veröffentlicht hat und in welchen Zeitschriften, wie viele Drittmittel sie eingeworben hat, zu welchen Konferenzen sie eingeladen wurde und von wem etc. (vgl. Jenkins 2013, 83). Akademisches Potential über diese Indikatoren auszubuchstabieren stellt bereits eine Verkürzung dar und feministische $\mathrm{Au}-$ torinnen weisen diese Verkürzung zurück, da sie annehmen, dass ein weites Verständnis (dem zufolge bspw. die Erhöhung der Diversität in einem Team oder pädagogisches Geschick auch als konstitutiv für akademisches Potential gelten) zu einem höheren Frauenanteil führen wird (vgl. Gheaus 2015, 171). An sich scheint mir ein weites Verständnis inhaltlich angebracht und auch ich glaube, dass es der Geschlechtergerechtigkeit förderlich wäre. Gerade deswegen werde ich aber im Weiteren mit dem skizzierten verkürzten Verständnis arbeiten, um mir meine argumentative Aufgabe nicht zu leicht zu machen. Ausgehend von diesem (verkürzten) Verständnis von akademischem Potential lässt sich die Position der Kritiker*innen folgendermaßen zusammenfassen:

(i) Die Einstellungskommission sollte die Bewerber*innen gemäß ihrem akademischen Potential für die zu besetzende Position in eine Rangordnung bringen und diejenige Bewerber*in auswählen, die am meisten akademisches Potential für die fragliche Position hat.

(ii) Falls es in diesem Ranking mehr als eine bestplatzierte Bewerber*in gibt, sollte die Einstellungskommission zwischen den bestplatzierten Bewerber*innen eine Münze werfen.

Im Folgenden nenne ich dies die traditionelle Sichtweise (TS).

Befürworter*innen einer schwachen Version der Praxis bevorzugter Anstellung $\left(\mathrm{PBA}_{\text {schwach }}\right.$ ) akzeptieren wie TS, dass die Einstellungskommission die Bewerber*in mit dem meisten akademischen Potential auswählen sollte. Aber gleichzeitig betonen sie, dass die Unterrepräsentation von Frauen in der akademischen Philosophie ein Problem darstellt, das Einstellungskommissionen adressieren sollten. Daher argumentieren sie für ein Vorgehen, dessen erster Schritt identisch ist mit dem ersten Schritt von TS, das aber in seinen Tie-Break-Regeln von TS abweicht: 
(i) Die Einstellungskommission sollte die Bewerber*innen gemäß ihrem akademischen Potential für die zu besetzende Position in eine Rangordnung bringen und diejenige Bewerber*in auswählen, die am meisten akademisches Potential für die fragliche Position hat.

(ii*) Falls es in dieser Rangordnung mehr als eine bestplatzierte Bewerber*in gibt und eine der Bestplatzierten eine Frau ist, sollte die Einstellungskommission die Bewerberin auswählen, die bestplatziert und weiblich ist.

(iii*) Falls es mehr als eine Bewerberin gibt, die bestplatziert und weiblich ist, sollte die Einstellungskommission zwischen den weiblichen Bestplatzierten eine Münze werfen.

Befürworter*innen einer starken Version der Praxis bevorzugter Anstellung $\left(\mathrm{PBA}_{\text {stark }}\right)$ vertreten eine radikalere Position. Zwar verneinen sie nicht rundheraus, dass die Einstellungskommission ihre Auswahl ausgehend vom akademischen Potential der Bewerber*innen treffen sollte. Aber für sie ist die Unterrepräsentation von Frauen in der akademischen Philosophie gravierend genug, um strengere Anforderungen an das Ranking-Verfahren zu rechtfertigen. Daher legen sie zuerst ein Mindestmaß an akademischem Potential als Schwellenwert fest, den Bewerber*innen für eine akademische Position überschreiten müssen, um für diese in die engere Auswahl zu kommen. Dieser Schwellenwert wird entweder relational festgelegt; in diesem Fall werden die Bewerber*innen zuerst ausgehend von ihrem akademischen Potential gerankt und dann wird festgesetzt, dass eine Kandidat*in zu den besten x Prozent dieser Rangordnung gehören muss, um in die engere Auswahl zu kommen. Alternativ kann der Schwellenwert absolut festgelegt werden; in diesem Fall werden bestimmte Merkmale festgelegt (wie etwa eine bestimmte Anzahl von Aufsätzen oder eine bestimmte Menge an eingeworbenen Drittmitteln), die eine Kandidat*in vorweisen muss, um im Rennen zu bleiben. Je nachdem, wie der Schwellenwert festgelegt wurde, werden unterschiedlich viele Kandidat*innen in die engere Auswahl kommen. Um zwischen diesen auszuwählen, greifen Befürworter*innen von PBA $_{\text {stark }}$ dann auf die letzte der folgenden Regeln zurück:

$\left(i^{* *}\right)$ Die Einstellungskommission sollte einen Schwellenwert an akademischem Potential für die zu besetzende Stelle festlegen, den eine Bewerber*in überschreiten muss, um in die engere Auswahl für die fragliche Stelle zu kommen.

(ii**) Falls es mehr als eine Bewerber*in gibt, die diesen Schwellenwert überschreitet, und eine von diesen eine Frau ist, sollte die Einstel- 
lungskommission diejenige Bewerberin auswählen, die den fraglichen Schwellenwert überschreitet und weiblich ist.

(iii**) Falls es mehr als eine Bewerberin gibt, die diesen Schwellenwert überschreitet und weiblich ist, sollte die Einstellungskommission zwischen den weiblichen Bewerberinnen, die den Schwellenwert überschreiten, eine Münze werfen.

Wie verhalten sich diese drei Positionen zueinander? Allen diskutierten Positionen zufolge sollte die Einstellungskommission bei ihrer Auswahl das akademische Potential der Bewerber*innen berücksichtigen. Doch TS und $\mathrm{PBA}_{\text {schwach }}$ sind der Auffassung, dass die Einstellungskommission die Kandidat*in mit dem meisten akademischen Potential auswählen sollte. Diese beiden Positionen vertreten hinsichtlich des akademischen Potentials der Bewerber*innen also eine Maximierungsthese. PBA $_{\text {stark }}$ begnügt sich dagegen damit zu fordern, dass die Einstellungskommission eine Kandidat*in auswählt, die genügend akademisches Potential mitbringt, wobei der festgelegte Schwellenwert fixiert, was genügend akademisches Potential ausmacht. Die beiden Versionen von PBA greifen aber nicht ausschließlich auf das akademische Potential der Kandidat*innen zurück, um eine Auswahl zu treffen, und vertreten daher keine Exklusivitätsthese. Stattdessen berücksichtigen sie auch die soziale Gruppenzugehörigkeit der Kandidat*innen, genauer gesagt ihr Geschlecht, um zwischen Bewerber*innen auszuwählen. Aber während $\mathrm{PBA}_{\text {schwach }}$ Geschlecht als Tie-Break-Kriterium zwischen den bestplatzierten Kandidat*innen verwendet, bringt PBA $_{\text {stark }}$ dieses Kriterium in Anschlag, um zwischen denjenigen Kandidat*innen zu entscheiden, die ausreichend akademisches Potential haben, um in die engere Auswahl zu kommen.

Der Vergleich zwischen TS und den beiden Versionen von PBA verdeutlicht die normativen Vorannahmen von TS: TS akzeptiert sowohl die Exklusivitäts- als auch die Maximierungsthese, $\mathrm{PBA}_{\text {schwach }}$ lehnt die Exklusivitätsthese ab, PBA $_{\text {stark }}$ dagegen sowohl die Exklusivitäts- als auch die Maximierungsthese. Der Blick auf diese Vorannahmen von TS zeigt also, wo der eigentliche Dissens zwischen TS und den beiden Versionen von PBA liegt. Im nächsten Abschnitt diskutiere ich, mit welchen Argumenten Kritiker*innen die Vorannahmen von TS verteidigen könnten, bevor ich dann in Abschnitt 4 für PBA argumentiere. Dabei werde ich mich auf die Verteidigung von PBA tark konzentrieren, da diese die Verteidigung von PBA $_{\text {schwach }}$ impliziert. 


\section{Das (meiner Ansicht nach) beste Argument für TS}

In der Einleitung hatte ich bereits erwähnt, dass es wenige Versuche gibt, TS explizit zu verteidigen. Die Kritiker*innen von PBA begnügen sich oft damit, die Argumente für diese Praxis zu widerlegen, anstatt eigene Argumente gegen sie vorzubringen. Ein Grund für diese Argumentationsstrategie könnte sein, dass TS in vielen Ländern schlicht das gängige Vorgehen ist und daher in praktischer Hinsicht keiner Begründung bedarf (zu dieser Einschätzung kommt auch Goldman 1975, 289). Aber aus den Überlegungen der Kritiker*innen lassen sich zwei Argumente für TS ableiten. Das erste baut auf Überlegungen zu Gleichbehandlung und Antidiskriminierung auf, das zweite auf Annahmen zum Zweck von Akademia. Im Folgenden stelle ich beide Argumente vor, wobei sich zeigen wird, dass nur das zweite zur Begründung für TS dienen kann.

Das erste Argument für TS behauptet, dass die Einstellungskommission die Bewerber*in mit dem meisten akademischen Potential für die fragliche Position auswählen sollte, weil sie nur so die Bewerber*innen als Gleiche ernst nimmt. Hinweise auf dieses Argument finden sich bspw. in Ronald Dworkins Diskussion von PBA. Denn nach Dworkin ist das stärkste Argument, das Kritiker*innen dieser Praxis vorbringen können, dass sie gegen das Recht des Individuums auf Gleichbehandlung durch das Gesetz verstößt (vgl. Dworkin 1977, 226). Eine ähnliche Überlegung scheint (zumindest an der Oberfläche) dem Rechtsstreit zugrunde zu liegen, den der konservative Aktivist Edward Blum gegen die Affirmative- action-Maßnahmen verschiedener amerikanischer Universitäten angestrengt hat. Denn PBA „falls afoul of our most basic civil rights principles“ nach Blum (vgl. Hartocollis und Saul 2017).

Die Gleichbehandlung, die durch TS verwirklicht wird, ist dabei die Gewährleistung von Chancengleichheit bei gleichem akademischen Potential. Denn aus TS folgt ja nicht, dass alle Bewerber*innen gleichermaßen einen Job bekommen oder dass die dritt- und die erstplatzierte Person die gleichen Chancen auf Einstellung haben. Aber da zwischen den gleichermaßen Bestplatzierten per Münzwurf entschieden werden muss, impliziert TS, dass Bewerber*innen mit gleichem akademischen Potential die gleiche Chance auf die ausgeschriebene Position haben. PBA unterläuft dagegen so verstandene Chancengleichheit (oder - wie Edwin Hettinger sagt - „the right of white males to an equal chance of employment“, Hettinger 1987, 46), da diese Praxis die Chancen auf eine akademische Position an das Geschlecht der Bewerber*innen koppelt, indem sie gebietet, bei mehreren Bestplat- 
zierten eine Frau auszuwählen. Wenn wir zudem der Auffassung sind, dass moralisch unzulässige Diskriminierung in ungerechtfertigter Schlechterstellung aufgrund vermeintlicher sozialer Gruppenzugehörigkeit besteht (wie bspw. Kasper Lippert-Rasmussen behauptet, vgl. Lippert-Rasmussen 2014, 45) und dass eine Verletzung der Chancengleichheit eine ungerechtfertigte Schlechterstellung darstellt, folgt aus der Tatsache, dass PBA nicht dem dargestellten Prinzip der Chancengleichheit entspricht, dass PBA eine Form von moralisch unzulässiger Diskriminierung darstellt: PBA räumt männlichen Mitbewerbern keine Chancengleichheit in Abhängigkeit von ihrem akademischen Potential ein, doch diese Ungleichbehandlung ist ausgehend vom Prinzip der Chancengleichheit bei gleichem akademischen Potential nicht zu rechtfertigen.

Gerade wenn es um die Verteilung von nicht lebensnotwendigen Gütern geht (und als solche sollten wir akademische Positionen - im Gegensatz zu Arbeitsplätzen im Allgemeinen - ansehen), ist das Prinzip der Chancengleichheit intuitiv plausibel. Deswegen spricht die Tatsache, dass TS als eine Umsetzung dieses Prinzips gelesen werden kann, durchaus für TS. Dennoch kann man TS nicht ausschließlich durch den Hinweis auf diesen Zusammenhang verteidigen, da dieser Hinweis allein TS' Maximierungsthese nicht austrägt. Es würde nämlich nicht gegen das Prinzip der Chancengleichheit bei gleichem akademischen Potential verstoßen, wenn die Einstellungskommission zwischen Gleichplatzierten per Münzwurf entscheidet, sich aber einfach nicht für eine der bestplatzierten Personen entscheidet. Nochmal anders gewendet: Aus der Vorschrift, Gleichplatzierten per Münzwurf gleiche Chancen auf den Job einzuräumen, folgt schlicht nicht, dass die Einstellungskommission die Bestplatzierte einstellen muss.

Da das erste Argument ungeeignet für die Begründung von TS’ Maximierungsthese ist, sollten wir uns dem zweiten und, wie mir scheint, überzeugenderen Argument für TS zuwenden. Diesem zufolge sollte die Einstellungskommission die Bewerber*in mit dem meisten akademischen Potential auswählen, weil sie so dem Zweck von Akademia am besten entspricht. Hettinger deutet ein solches teleologisches Argument an, wenn er behauptet, dass sich die Kritiker*innen gegen PBA aussprechen, weil die Bestplatzierte auszuwählen die Effizienz von Akademia befördert (vgl. Hettinger 1987, 43f. sowie Pojman 1998, 111). Die Rede von Effizienz setzt nämlich, ebenso wie der gelegentlich gehörte Hinweis, dass man das Geld der Steuerzahler*innen nicht für PBA verschwenden sollte, einen Zweck von Akademia voraus. Denn ineffizient handeln bzw. Geld verschwenden kann man nur, wenn es einen 
Zweck gibt, der in maximal effizienter Form befördert werden sollte. Doch was könnte der Zweck von Akademia sein? Zur Ausrichtung von TS passt am besten die (auch an sich intuitive) Annahme, dass der Zweck von Akademia darin besteht, neue Erkenntnisse hervorzubringen. Wenn man dies als Zweck von Akademia voraussetzt, können wir folgendes teleologische Argument für TS zusammenstellen:

(1) Der Zweck von Akademia ist, neue Erkenntnisse hervorzubringen.

(2) Akademische Institutionen sollten ihre Ressourcen so verteilen, dass sie diesem Zweck am besten entsprechen.

(3) Akademische Positionen sind Teil der Ressourcen, die akademische Institutionen zu verteilen haben.

(4) Deswegen sollten akademische Institutionen ihre Ressourcen so verteilen, dass sie am besten neue Erkenntnisse hervorbringen.

(5) Um am besten neue Erkenntnisse hervorzubringen, sollten akademische Institutionen nur diejenigen Merkmale der Bewerber*innen berücksichtigen, die relevant sind für die Hervorbringung neuer Erkenntnisse.

(6) Das einzige Merkmal der Bewerber*innen, das relevant für die Hervorbringung neuer Erkenntnisse ist, ist ihr akademisches Potential.

(7) Je mehr akademisches Potential eine Bewerber*in hat, umso besser ist sie in der Hervorbringung neuer Erkenntnisse.

(K1) Deswegen sollten akademische Institutionen nur das akademische Potential der Bewerber*innen berücksichtigen.

(K2) Deswegen sollten akademische Institutionen akademische Positionen an die Bewerber*in mit dem meisten akademischen Potential verteilen.

Anders als die Überlegungen zu Gleichbehandlung und Antidiskriminierung, die dem ersten Argument zugrunde lagen, unterfüttert dieses Argument TS passgenau, da (K1) und (K2) nichts anderes sind als TS' Exklusivitäts- und Maximierungsthese. Um zu zeigen, wie diese beiden Konklusionen aus Obigem folgen, führe ich kurz die einzelnen Prämissen aus. (1) artikuliert die teleologische Annahme, dass der Zweck von Akademia in der Hervorbringung neuer Erkenntnisse liegt. (2) ist TS' tragende normative Annahme. (3) ist eine deskriptive Annahme, wonach akademische Positionen Teil der Ressourcen sind, über die akademische Institutionen verfügen. Andere Ressourcen wären bspw. Forschungsgelder oder Vortragseinladungen. (4) folgt aus (1), (2) und (3). (5) ist eine prudentielle Annahme darüber, wie akademische Institutionen bei der Bewertung von Bewerber*innen vorgehen müssen, um 
ihrem Zweck am besten gerecht zu werden. (6) ist eine deskriptive Annahme dazu, welche Merkmale einer Bewerber*in ausschlaggebend dafür sind, dass sie erfolgreich neue Erkenntnisse hervorbringt. (7) ist eine weitere deskriptive Annahme dazu, wie akademisches Potential und die Hervorbringung neuer Erkenntnisse zusammenhängen. (K1) folgt aus (4), (5) und (6), (K2) dagegen aus (4), (6) und (7). Damit ist dieses zweite Argument schlüssig und, zumindest im Vergleich zum vorher diskutierten, das bessere Argument für TS. Wenn wir TS kritisieren möchten, sollten wir uns also mit diesem zweiten Argument auseinandersetzen.

Und natürlich gäbe es viel zu den einzelnen Prämissen zu sagen. ${ }^{4}$ (3) und (5) scheinen mir unstrittig. (1) dagegen ist angreifbar, denn warum sollten akademische Institutionen nur einem einzigen Zweck gerecht werden? Ebenso angreifbar sind (6) und, in Abhängigkeit davon, (7), insbesondere wenn man in Rechnung stellt, dass wir akademisches Potential häufig nur über Indikatormerkmale ermitteln (dies ist die Kritik feministischer Autorinnen, die ich bereits am Beginn von Abschnitt 2 erwähnt hatte). Im Folgen-

$4 \quad$ Meiner Ansicht nach kann man Thomas Nagels Argument für PBA ebenso wie Beate Rösslers und Martina Herrmanns als Angriffe auf (1) verstehen. Denn die Autor*innen rechtfertigen eine mit $\mathrm{PBA}_{\text {stark }}$ unter Umständen einhergehende Einbuße an Erkenntnisgewinn durch den Hinweis, dass so andere wichtige Güter befördert werden können. Nagel hat dabei die Besserstellung von bisher schlechter Gestellten im Sinn (vgl. Nagel 1973, 362), Rössler ein höheres Maß an sozialer Gleichheit (vgl. Rössler 1999, 46) und Herrmann die verbesserte Partizipation von Frauen (vgl. Herrmann 1999, 97). Ihnen zufolge ist Akademia also nicht nur auf die Umsetzung eines Zieles festgelegt. Marilyn Friedmans Überlegungen kann man dagegen als Zurückweisung von (6) verstehen. Denn Friedman betont, dass Diversität epistemisch, d.h. bei der Hervorbringung neuer Erkenntnisse hilfreich sein kann (vgl. Friedman 2013, 34f.). Da der Potential-Begriff, der (6) zugrunde liegt, diesem Faktum nicht Rechnung trägt, muss also (6) zurückgewiesen werden. Das Kompensationsargument, eines der einflussreichsten Argumente für PBA, ist dagegen mit allen Prämissen von TS einverstanden. Nach diesem Argument stellt TS an sich richtig dar, wie akademische Positionen unter idealen Bedingungen zu verteilen wären; weil es aber bei früheren Verteilungen zu Ungerechtigkeiten gekommen ist, sollten jetzt die vorher Benachteiligten durch $\mathrm{PBA}_{\text {stark }}$ kompensiert werden (vgl. Thomson 1973, 383). Gegen das Kompensationsargument wurde oft eingewendet, dass $\mathrm{PBA}_{\text {stark }}$ nicht gewährleistet, dass gerade diejenigen Kompensation leisten müssen, die vorher ungerechtfertigt bevorzugt wurden. Diese Kritik ist richtig, zudem räumt das Kompensationsargument TS zu viel Geltung ein. Denn wie ich zeigen werde, sollten wir TS so nicht akzeptieren. 
den akzeptiere ich jedoch (1), (6) und (7) und begnüge mich damit, kritisch auf (2) einzugehen.

\section{Warum die traditionelle Sichtweise und die Praxis bevorzugter Anstellung miteinander vereinbar sind}

Gegen dieses beste Argument für TS und insbesondere gegen (2) werde ich nun Folgendes einwenden: Zum einen akzeptieren wir nicht TS' tragende normative Prämisse (2), sondern nur eine abgeschwächte Version ( $2^{*}$ ). Doch wenn man TS auf der abgeschwächten Prämisse $\left(2^{*}\right)$ aufbaut, ist TS kompatibel mit $\mathrm{PBA}_{\text {stark }}$ (für eine ähnliche Vorgehensweise vgl. Meshelski 2016). Zum anderen setzt $\mathrm{PBA}_{\text {stark }}$, ausgehend von bestimmten Annahmen über die akademische Philosophie in Deutschland, die moralischen Erwägungen, welche hinter TS stehen, besser um als TS selbst. Das heißt, wenn wir TS richtig (nämlich in ihrer abgeschwächten Form) verstehen, spricht diese Sichtweise also nicht grundsätzlich gegen $P B A_{\text {stark }}$, und ausgehend von bestimmten Annahmen über die akademische Philosophie in Deutschland sollten die Anhänger*innen von TS selbst für PBA ${ }_{\text {stark }}$ eintreten.

Um diese Behauptungen einzuholen, muss ich in einem ersten Schritt zeigen, dass wir nur eine abgeschwächte Version von (2) akzeptieren. Prima facie erscheint dies nicht als erfolgversprechendes Unterfangen. Denn (2) behauptet nur, dass akademische Institutionen die Ressourcen, die ihnen zur Verfügung stehen, so verteilen sollten, dass sie ihrem Zweck am besten entsprechen. Akademische Institutionen sind letztlich nichts anderes als Artefakte, d.h. Entitäten, die von Menschen zu einem bestimmten Zweck erschaffen wurden, und wie bei allen Artefakten liegt es nahe anzunehmen, dass sie - eben weil sie zur Erfüllung eines bestimmten Zwecks geschaffen wurden - darauf festgelegt sind, diesem Zweck besonders gut nachzukommen. Auf den ersten Blick scheint es also, als würde (2) keiner weiteren Begründung, geschweige denn einer Abschwächung bedürfen, sondern wäre trivialerweise wahr.

Eine mögliche Antwortstrategie hierauf wäre grundsätzlich zu hinterfragen, ob Artefakte wirklich darauf festgelegt sind, ihren Zwecken besonders gut nachzukommen (für eine kritische Auseinandersetzung mit dieser These vgl. Bratu und Dittmeyer 2016, Abschnitt 3). Für meine argumentativen Belange reicht es aber an dieser Stelle aus darauf hinzuweisen, dass wir zumindest mit Blick auf das Artefakt akademische Institution zu einer Abschwächung bereit sind. Denn in Deutschland akzeptieren wir, dass aka- 
demische Institutionen ihre Ressourcen nicht immer so verteilen, dass sie in optimaler Weise neue Erkenntnisse hervorbringen, und also gelegentlich ihrem Zweck nicht optimal nachkommen. So formuliert bspw. die Deklaration von Helsinki, an der sich medizinische Forschung in Deutschland orientieren muss, strenge Auflagen für den Umgang mit bzw. die Forschung an lebenden Personen (vgl. WMA), und das Stammzellgesetz macht ähnlich stringente Vorschriften für den wissenschaftlichen Umgang mit menschlichem biologischen Material (insbesondere mit embryonalen Stammzellen, vgl. StZG). Ebenso ist die Forschung an Tieren durch das Tierschutzgesetz weitgehend geregelt, und die Forschung an bestimmten Tierarten (etwa an Primaten) ist sogar prinzipiell verboten (vgl. TierSchG). Die genannten Gesetze schreiben also fest, dass akademische Ressourcen wie Forschungsgelder nicht für bestimmte Projekte eingesetzt werden dürfen. Doch diese Einschränkungen gelten nicht deswegen, weil diese Projekte dem Zweck von Akademia zuwiderlaufen würden - gerade von der Stammzellenforschung erhoffen sich viele Wissenschaftler*innen innovative und potentiell lebensrettende Erkenntnisse. Vielmehr tragen diese Einschränkungen moralischen Erwägungen Rechnung, d.h., sie zeigen, dass wir gewillt sind, den Zweck, auf den Akademia ausgerichtet ist, mit anderen moralischen Erwägungen (wie etwa dem Wohle der Patient*innen oder der Vermeidung von Leid) zu vergleichen und letztere stärker zu gewichten. Die in Deutschland etablierte akademische Praxis zeigt also, dass wir gewillt sind, (2) zu ( $\left.2^{*}\right)$ abzuschwächen: (2*) Akademische Institutionen sollten ihre Ressourcen so verteilen, dass sie diesem Zweck (nämlich: der Hervorbringung neuer Erkenntnisse) am besten entsprechen, es sei denn, gewichtige moralische Erwägungen sprechen dagegen.

Meiner Ansicht nach sollte die Anhänger*in von TS (2*) als Abschwächung von (2) akzeptieren. Denn $\left(2^{*}\right)$ entspricht nicht nur der deutschen Wissenschaftspraxis, sondern trägt zudem der Intuition Rechnung, dass Wissenschaft nicht im moralischen Vakuum geschieht und dass wir demnach sicherstellen müssen, dass sie nicht gegen das verstößt, was wir uns wechselseitig schulden. Es wäre unfair anzunehmen, dass Kritiker*innen von PBA für moralische Erwägungen gänzlich taub sind und um jeden Preis darauf beharren, dass akademische Institutionen den Erkenntnisgewinn maximieren. Eigentlich sollte die Kritiker*in also mit (2*) arbeiten und (4) sowie, daraus resultierend, (K1) und (K2) folgendermaßen abschwächen: 
$\left(4^{*}\right)$ Deswegen sollten akademische Institutionen ihre Ressourcen so verteilen, dass sie am besten neue Erkenntnisse hervorbringen, es sei denn, gewichtige moralische Erwägungen sprechen dagegen.

$\left(\mathrm{K}^{*}{ }^{*}\right)$ Deswegen sollten akademische Institutionen nur das akademische Potential der Bewerber*innen berücksichtigen, es sei denn, gewichtige moralische Erwägungen sprechen dagegen.

$\left(\mathrm{K}_{2}{ }^{*}\right)$ Deswegen sollten akademische Institutionen akademische Positionen an die Bewerber*in mit dem meisten akademischen Potential verteilen, es sei denn, gewichtige moralische Erwägungen sprechen dagegen.

Doch in dieser Version ist die Position der Kritiker*in mit PBA ${ }_{\text {stark }}$ kompatibel. Zur Erinnerung: PBA $_{\text {stark }}$ lehnte zum einen die Exklusivitätsthese (K1) ab und behauptete also, dass es zulässig ist, das Geschlecht der Bewerber*innen als Tie-Break-Kriterium zu berücksichtigen, wenn dadurch eine Frau angestellt wird. Zum anderen wies PBA $_{\text {stark }}$ die Maximierungsthese (K2) zurück und vertrat, dass es erlaubt ist, eine Bewerberin anzustellen, die genügend (aber eben nicht am meisten) akademisches Potential für eine Position mitbringt, wenn sie weiblich ist. In ihren abgeschwächten Versionen schließen nun weder $\left(\mathrm{K}^{*}\right)$ noch $\left(\mathrm{K}^{*}\right)$ grundsätzlich aus, was $\mathrm{PBA}_{\text {stark }}$ fordert. Denn sie räumen ein, dass es zulässig ist, nicht ausschließlich das akademische Potential der Bewerber*innen zu berücksichtigen bzw. dieses nicht zu maximieren, wenn hierfür gewichtige moralische Erwägungen sprechen. Damit ist gezeigt, dass TS mit $\mathrm{PBA}_{\text {stark }}$ vereinbar sein kann.

Um zu zeigen, dass TS mit $\mathrm{PBA}_{\text {stark }}$ vereinbar ist, muss ich in einem zweiten Schritt darlegen, dass tatsächlich gewichtige moralische Erwägungen dafür sprechen, in der akademischen Philosophie in Deutschland zugunsten von weiblichen Bewerberinnen von der Exklusivitäts- und Maximierungsthese abzugehen. Um den Kritiker*innen von PBA entgegenzukommen, greife ich an dieser Stelle auf Erwägungen zurück, die bei der Verteidigung von TS bereits eine Rolle gespielt haben. Denn ich behaupte, dass die Anwendung von TS unter bestimmten Umständen nicht verlässlich dem Prinzip der Chancengleichheit bei gleichem Potential entspricht und zudem die Menge verfügbaren akademischen Potentials einschränkt. Unter denselben Umständen würde die Durchführung von PBA $_{\text {stark }}$ dagegen auf Dauer die Konformität unserer Stellenverteilung mit dem genannten Prinzip der Chancengleichheit sicherstellen und zudem die Menge verfügbaren akademischen Potentials vergrößern. Sollten die fraglichen Umstände in der deutschen akademischen Philosophie gegeben sein, sollten die Anhänger*innen 
von TS also aus denselben Gründen, die in ihren Augen für TS sprachen, stattdessen für die Durchführung von $\mathrm{PBA}_{\text {stark }}$ sein. Diese Behauptung werde ich nun Stück für Stück einholen.

Die Umstände, die meine Behauptung voraussetzt, sind dabei die Existenz und weite Verbreitung von so genannten impliziten Vorurteilen (implicit biases) innerhalb einer akademischen Disziplin. Solche Vorurteile sind zu erwarten, wenn die Mitglieder einer sozialen Gruppe in einer Disziplin als atypisch betrachtet werden, entweder weil nur wenige von ihnen darin tätig sind oder weil es (explizite oder implizite) soziale Narrative gibt, die sie als atypisch für diese Disziplin kennzeichnen. Gegenstand solcher Vorurteile zu sein ist für die Betroffenen oft von Nachteil. So werden sie häufiger von anderen Mitgliedern ihrer Disziplin als Außenseiter behandelt und müssen bspw. sich selbst und ihre Arbeit häufiger rechtfertigen (vgl. Leuschner 2019, 240) - Verhaltensweisen, die als Mikroaggressionen wahrgenommen werden können. Zudem werden ihre Leistungen oft, und zwar vor allem dann, wenn es schnell gehen muss, schlechter bewertet als eigentlich angebracht (vgl. Saul 2013, 40f.). Diese direkten Effekte uneingestandener Vorurteile ziehen indirekte Effekte nach sich, da sie die Betroffenen verunsichern und zu Selbstzweifeln an der eigenen Leistung und Befähigung führen. Solche Zweifel erschweren es den Betroffenen dann wiederum in stressigen Prüfungssituationen ihr Wissen und Können erfolgreich abzurufen (ein Phänomen, das als stereotype threat bezeichnet wird, vgl. Saul 2013, 41f.) oder führen zu risikoaversem Verhalten wie etwa sehr lange an Forschungsarbeiten zu feilen, diese gar nicht erst zur Begutachtung einzureichen oder sich von Anfang an auf weniger prestigeträchtige Aufgaben zu beschränken. Letztlich entsteht so der Teufelskreis einer selbsterfüllenden Prophezeiung (vgl. Leuschner 2019, 242): Uneingestandene Vorurteile führen zu einem unangenehmen Arbeitsumfeld und zu unfairen Bewertungen; diese erschweren es den Betroffenen, ihr Potential zu präsentieren oder überhaupt zu entwickeln; dies bestätigt wiederum den Außenseiterstatus der betroffenen Gruppe und damit letztlich die uneingestandenen Vorurteile ihren Mitgliedern gegenüber.

Unter Umständen wie den skizzierten entspricht die Anwendung von TS nicht verlässlich dem Prinzip der Chancengleichheit bei gleichem akademischen Potential. Denn zwischen Gleichplatzierten per Münzwurf zu entscheiden stellt nur dann sicher, dass gleiches akademisches Potential gleiche Einstellungschancen impliziert, wenn das zugrundeliegende Ranking das akademische Potential der Bewerber*innen korrekt widerspiegelt. Doch einer der direkten Effekte uneingestandener Vorurteile ist ja gerade, 
dass deren Leistungen oft nicht angemessen bewertet werden. Die Durchführung von Affirmative-action-Maßnahmen wie $\mathrm{PBA}_{\text {stark }}$ könnte hier auf Dauer Abhilfe schaffen. Denn eine Strategie zum Abbau uneingestandener Vorurteile besteht in so genannter exposure und also darin, die Mitglieder der fraglichen Disziplin mit Eindrücken von und Erfahrungen mit Mitgliedern marginalisierter Gruppen zu konfrontieren, die den (impliziten und expliziten) sozialen Narrativen zuwiderlaufen, die ihren Vorurteilen zugrunde liegen (vgl. Lai et al. 2014, 16f. und 45). Dies ist allerdings nur zielführend, wenn ausreichend Gegenbeispiele zu den problematischen Narrativen bereitgestellt werden (denn die die Narrative widerlegenden Einzelfälle werden scheinbar eher als Ausnahmen wahrgenommen, die die Regel bestätigen). Durch PBA ${ }_{\text {stark }}$ könnte man den Betroffenen in richtigem Maße Sichtbarkeit verschaffen. Würden dadurch die Narrative, die ihnen Außenseiterstatus bescherten, effektiv unterwandert, könnte dies auf die Dauer u.a. bei der Vergabe akademischer Positionen zu Rangordnungen der Kandidat*innen führen, die das akademische Potential aller Beteiligten angemessen widerspiegeln, und so sicherstellen, dass die Anwendung von TS tatsächlich Chancengleichheit bei gleichem Potential impliziert. Zudem könnte die Durchführung von PBA $_{\text {stark }}$ die Betroffenen dabei unterstützen, ihr akademisches Potential erfolgreicher zu präsentieren bzw. überhaupt erst zu entwickeln, da sie nicht mehr kontinuierlich dazu gebracht würden an sich selbst zu zweifeln. Dies würde die Menge des in der fraglichen Disziplin vorhandenen akademischen Potentials vergrößern und wäre insofern förderlich für die Hervorbringung neuer Erkenntnisse. Wenn wir annehmen (wofür ich in 3 argumentiert habe), dass Anhänger*innen von TS für diese Verteilungsregel eintreten, weil es ihnen um Chancengleichheit bei gleichem akademischen Potential geht und weil sie wollen, dass Akademia möglichst viele neue Erkenntnisse hervorbringt, sollten sie also immer dann, wenn in einer akademischen Disziplin uneingestandene Vorurteile verbreitet sind, eigentlich für die Durchführung von PBA $_{\text {stark }}$ eintreten.

Um diese Konklusion auf die deutsche akademische Philosophie zu übertragen, müsste ich abschließend zeigen, dass diese von uneingestandenen Vorurteilen gegenüber Frauen gekennzeichnet ist. Dies ist eine Aufgabe für die empirisch arbeitenden Wissenschaften, die ich nicht übernehmen kann. Doch meiner Ansicht nach gibt es gute Gründe hiervon auszugehen. Zum einen sind die Bedingungen für Vorurteile gegenüber Frauen als Philosophinnen gegeben. Denn Frauen sind in der deutschen akademischen Philosophie tatsächlich insofern atypisch, als es, wie in der Einleitung darge- 
legt, auf den höheren Stufen der akademischen Hierarchieleiter nur wenige Frauen gibt. Zudem verbinden Vertreter*innen des Fachs Philosophie ihre Disziplin mit Attributen wie etwa angeborener Genialität (vgl. Leslie et al. 2015, 264) oder der Fähigkeit zu logischem bzw. abstraktem Denken und zu Rationalität (vgl. di Bella et al. 2016, 301), welche nach wie vor männlich konnotiert sind. Zum anderen würde die Annahme, dass es solche Vorurteile gibt und dass sie verbreitet sind, zumindest teilweise die Mikroaggressionen, von denen Frauen in der Philosophie immer wieder berichten (vgl. Brennan 2013), ebenso erklären wie ihr risikoaverses Publikationsverhalten (vgl. Leuschner 2019, 243) sowie das Phänomen der leaky pipeline insgesamt (vgl. Antony 2012; Leuschner 2015, 63; widersprechen würden Sesardic und de Clerq 2014).

Wenn wir annehmen, dass die deutsche akademische Philosophie von uneingestandenen Vorurteilen gegenüber Frauen gekennzeichnet ist, zeigt sich, dass TS nicht nur grundsätzlich, sondern tatsächlich mit $\mathrm{PBA}_{s-}$ tark kompatibel ist. Denn dann sprechen gewichtige moralische Erwägungen dagegen, auf Kosten weiblicher Bewerberinnen an der Exklusivitäts- und Maximierungsthese in ihrer ursprünglichen Form festzuhalten. Bei diesen Erwägungen handelt es sich gerade um diejenigen, die auch Vertreter*innen von TS anführen würden, nämlich um Erwägungen zur Chancengleichheit und zur Mehrung akademischer Erkenntnisse. Ausgehend von dieser Annahme trägt $\mathrm{PBA}_{\text {stark }}$ diesen Erwägungen besser Rechnung als TS selbst. Insofern sollten Anhänger*innen von TS für $\mathrm{PBA}_{\text {stark }}$ eintreten.

\section{Ein möglicher Einwand?}

Eine Anhänger*in von TS könnte zugestehen, dass es in der deutschen akademischen Philosophie uneingestandene Vorurteile gegen Frauen gibt, so dass die Anwendung von TS deren Chancengleichheit bei gleichem akademischen Potential nicht verlässlich sicherstellen kann. Sie könnte zudem zugestehen, dass dies durch die Durchführung von $\mathrm{PBA}_{\text {stark }}$ auf Dauer verbessert würde. Und dennoch könnte sie bestreiten, daher auf die Durchführung von $\mathrm{PBA}_{\text {stark }}$ festgelegt zu sein und zwar weil $\mathrm{PBA}_{\text {stark }}$ kein moralisch zulässiges Mittel sei, diesen besseren Zustand herbeizuführen. Denn die Durchführung von PBA $_{\text {stark }}$ impliziere, dass männliche Bewerber aufgrund ihres Geschlechts nicht angestellt würden, und dies sei unzulässig diskriminierend (vgl. Newton 1973, 310 sowie Gräfrath 1999, 61. Anita Allen scheint etwas Ähnliches anzunehmen, da es ihr sinnvoll erscheint, dass sich Personen, die von PBA 
profitiert haben, bei denjenigen, die davon benachteiligt wurden, entschuldigen, vgl. Allen 2011). Denselben Zweifel könnte die Vertreter*in auch durch die Behauptung zum Ausdruck bringen, dass meinem Argument ein falsches Verständnis von Chancengleichheit zugrunde liegt. Denn mein Argument behandelt Chancengleichheit als Wert, den es umzusetzen gilt und zwar so umfassend wie möglich. Doch eigentlich sollte man Chancengleichheit als side constraint betrachten: Auch wenn ein Weltzustand, in dem Chancengleichheit verlässlich umgesetzt wird, moralisch besser sei als einer, in dem dies nicht der Fall ist, sei es dennoch moralisch unzulässig, diesen Weltzustand herbeizuführen, wenn man dafür punktuell gegen Chancengleichheit verstoßen muss. Und genau dies geschähe, so die Anhänger*in von TS, würden wir $\mathrm{PBA}_{\text {stark }}$ anwenden.

Meiner Ansicht nach kann dieser Einwand nicht überzeugen, da er von einer Annahme ausgeht, welche die Anhänger*in von TS an dieser Stelle nicht mehr voraussetzen darf, nämlich von der Exklusivitätsthese in ihrer nicht abgeschwächten Form (K1). Denn die Anhänger*in muss ihre Annahme, dass es ungerecht ist, wenn männliche Bewerber aufgrund ihres Geschlechts nicht angestellt werden, begründen. Die naheliegende Begründung hierfür ist die These, dass bei der Verteilung akademischer Positionen nur das akademische Potential der Bewerber*innen berücksichtigt werden sollte. Doch dies ist nichts anderes als (K1) und also eine These, von der ich oben gezeigt habe, dass wir sie zumindest für die akademische Praxis in Deutschland nicht in dieser unqualifizierten Art akzeptieren.

In der dargestellten Form greift der Einwand der Anhänger*in von TS also nicht. Doch er lässt sich verbessern, wenn man ihm statt (K1) die abgeschwächte Version (K1*) zugrunde legt. In dieser modifizierten Version würde die Anhänger*in von TS lediglich behaupten, dass in dem hier diskutierten Fall - also bei der Verteilung von Positionen in der akademischen Philosophie in Deutschland - keine gewichtigen moralischen Erwägungen dafür sprechen, auch andere Merkmale der Bewerber*innen zu berücksichtigen als deren akademisches Potential. PBA stark $_{\text {äre }}$ demnach nicht grundsätzlich ein moralisch unzulässiges Mittel, um einen besseren Zustand herbeizuführen, sondern nur in diesem konkreten Fall. Um diese These zu begründen, könnte die Anhänger*in von TS entweder (a) moralphilosophische Erwägungen anführen und bspw. behaupten, dass ein Zustand, der verlässlich Chancengleichheit garantiert, insgesamt nicht besonders wünschenswert ist oder zumindest nicht wünschenswert genug, um die Einbuße an Erkenntnissen aufzuwiegen, die Anhänger*innen von TS 
von der Durchführung von PBA $_{\text {stark }}$ erwarten. Oder sie könnte (b) auf faktischer Ebene leugnen, was ich oben dargelegt habe, nämlich dass uneingestandene Vorurteile die akademische Philosophie in Deutschland kennzeichnen.

(a) erscheint mir in beiden skizzierten Varianten keine erfolgreiche Argumentationsstrategie. Zum einen ist uns Chancengleichheit bei der Verteilung nicht lebensnotwendiger Güter durchaus wichtig. Vor allem aber ist unklar, ob PBA $_{\text {stark }}$ wirklich zu einer derart hohen Einbuße an Erkenntnissen führt, dass es insgesamt besser erscheint, auf einen dauerhaften Zugewinn an Chancengleichheit zu verzichten. Grundsätzlich hat die Anhänger*in von TS zwar recht mit dem Hinweis, dass die moralische Abwägung zwischen Erkenntnisgewinn und Chancengleichheit auch zugunsten von Ersterem ausgehen kann. Wäre bspw. abzusehen, dass PBA ${ }_{\text {stark }}$ die Anstellung von Personen mit so gut wie gar keinem akademischen Potential zur Folge hätte, spräche die (daraus wahrscheinlich resultierende) Verarmung der akademischen Philosophie wohl gegen $\mathrm{PBA}_{\text {stark }}$. Aber natürlich steht uns offen, $\mathrm{PBA}_{\text {stark }}$ so zu gestalten, dass diese Folge vermieden werden kann. Dafür müssen wir lediglich sicherstellen, dass der Schwellenwert, den PBA $_{\text {stark }}$ vorsieht, nicht zu niedrig festgelegt wird, so dass die Bewerber*innen, die ihn überschreiten und in die engere Auswahl kommen, genug akademisches Potential mitbringen, auf dass wir von ihnen einen ausreichenden Beitrag zum Erkenntnisgewinn erhoffen dürfen. Das heißt, wir können den moralphilosophischen Einwand der Anhänger*in von TS entkräften, indem wir sicherstellen, dass der von $\mathrm{PBA}_{\text {stark }}$ geforderte Schwellenwert nur Personen mit ausreichend akademischem Potential in die engere Auswahl kommen lässt.

Letztlich ist daher (b) die erfolgversprechendere Argumentationsstrategie für die Anhänger*in von TS. In der Theorie erscheint mir diese Strategie plausibel. Denn so, wie ich $\mathrm{PBA}_{\text {stark }}$ eingeführt und verteidigt habe, ist diese Praxis eine Ausgleichsstrategie, die sicherstellen soll, dass unsere Verteilung akademischer Positionen auf Dauer dem Ideal der Chancengleichheit entspricht. Wenn dies bereits erfüllt ist, ist kein weiterer Ausgleich notwendig. Hieraus folgt auch, wo in der Theorie die Grenze für $\mathrm{PBA}_{\text {stark }}$ liegt, d.h., ab wann diese Praxis ungerechtfertigt ist, nämlich genau dann, wenn verzerrende Phänomene wie uneingestandene Vorurteile und stereotype threat keine Rolle mehr bei unserer Verteilung akademischer Positionen spielen, weil Frauen in der akademischen Philosophie genug Sichtbarkeit haben, als dass sie nicht mehr als atypisch angesehen werden. Sobald dies gegeben ist, kann TS ohne weitere Einschränkungen durchge- 
führt werden. ${ }^{5}$ Doch wie ich oben dargelegt habe, gibt es meiner Ansicht nach Grund zur Annahme, dass wir in der Praxis der akademischen Philosophie in Deutschland noch nicht an diesem Punkt angekommen sind. Ich gestehe mit anderen Worten zu, dass (b) eine erfolgversprechende Argumentationsstrategie gegen $\mathrm{PBA}_{\text {stark }}$ wäre, wenn die Startprämisse dieser Strategie zutreffen würde - doch es erscheint mir zweifelhaft, dass die akademische Philosophie in Deutschland bereits frei von den genannten Verzerrungen ist.

\section{Schluss: Mögliche Erweiterungen?}

Mein Argument dafür, dass die bevorzugte Anstellung von Frauen in der akademischen Philosophie in Deutschland moralisch zulässig ist, beruhte auf zwei Annahmen: dass es zulässig ist, teilweise vom Zweck akademischer Institutionen abzugehen, wenn gewichtige moralische Erwägungen hierfür sprechen; und dass in der akademischen Philosophie in Deutschland Phänomene am Werk sind, die dazu führen, dass die Vergabe von Positionen aktuell nicht dem Ideal der Chancengleichheit entspricht. Die Möglichkeit von PBA wird aber natürlich nicht nur für die akademische Philosophie in Deutschland diskutiert, sondern sowohl für andere akademische Disziplinen als auch für andere Länder, vor allem aber für die Vergabe von Jobs und Positionen auf dem nichtakademischen Arbeitsmarkt. Letzteres ist in meinen Augen die viel dringendere Frage, weil sie deutlich mehr Menschen betrifft, doch leider lassen sich meine Überlegungen nicht ohne Weiteres auf diese Frage übertragen. Vielmehr muss man zeigen, dass die beiden soeben genannten Annahmen auch für den nichtakademischen Arbeitsmarkt gelten. Diese Arbeit kann ich in diesem Artikel nicht leisten, doch ich bin optimistisch, dass dies möglich ist. Denn grundsätzlich sind wir bereit, den Markt an moralischen Erwägungen auszurichten, und leider gibt es auch im Bereich der freien Marktwirtschaft Narrative über bestimmte soziale Gruppen, die diese als atypisch erscheinen lassen und also Raum für Diskriminierung geben.

5 Personen, die nicht Prämisse (2), sondern Prämisse (1) von TS zurückweisen, würden dies bestreiten und noch weitere Einschränkungen für die Durchführung von TS fordern. Diese Strategie scheint mir an sich auch sinnvoll, ist aber nicht diejenige, die ich in diesem Text verfolgt habe. 


\section{Literatur}

Allen, Anita. 2011. „Was I Entitled or Should I Apologize? Affirmative Action Going Forward“. Journal of Ethics 15 (3), 253-263.

Antony, Louise. 2012. „Different Voices or Perfect Storm: Why Are There So Few Women in Philosophy?“. Journal of Social Philosophy 43 (3), 227-255.

Arneson, Richard. 2000. „Luck egalitarianism and prioritarianism“. Ethics 110 (2), 339-349.

Baron, Sam, Tom Dougherty, und Kristie Miller. 2015. „Why is there female under-representation among philosophy majors? Evidence of a pre-university effect“. Ergo 2 (14), 329-365.

BayHSchG (Bayrisches Hochschulgesetz): http://www.gesetze-bayern.de/Content/ Document/BayHSchG (zuletzt abgerufen am 26.5.2019).

Di Bella, Laura, Eleanor Miles, und Jennifer Saul. 2016. „Philosophers Explicitly Associate Philosophy with Maleness: An Examination of Implicit and Explicit Gender Stereotypes in Philosophy“. In Implicit Bias and Philosophy. Volume 1: Metaphysics and Epistemology, herausgegeben von Michael Brownstein und Jennifer Saul, 283-308. Oxford et al.: Oxford University Press.

Bratu, Christine und Moritz Dittmeyer. 2016. „Constitutivism about Practical Principles: Its Claims, Goals, Task and Failure“. Philosophia 44 (4), 1129-1143.

Brennan, Samantha. 2013. „Rethinking the Moral Significance of Micro-Inequities: The Case of Women in Philosophy“. In Women in Philosophy: What Needs to Change?, herausgegeben von Katrina Hutchison und Fiona Jenkins, 180-196. Oxford et al.: Oxford University Press.

Dworkin, Ronald. 1977. „Reverse Discrimination“. In Taking Rights Seriously, herausgegeben von Ronald Dworkin, 223-239. Cambridge MA: Harvard University Press.

Friedman, Marilyn. 2013. „Women in Philosophy: Why Should We Care?“. In Women in Philosophy: What Needs to Change?, herausgegeben von Katrina Hutchison und Fiona Jenkins, 21-38. Oxford et al.: Oxford University Press.

Fullinwider, Robert. 2018. „Affirmative Action“. In The Stanford Encyclopedia of Philosophy, herausgegeben von Edward Zalta, https://plato.stanford.edu/archives/sum2018/entries/affirmative-action/ (zuletzt abgerufen am 14.10.2019).

Gheaus, Anca. 2015. „Three Cheers for the Token Women!“. Journal of Applied Philosophy, 32 (2), 163-176.

Goldman, Alan. 1975 „Limits to the Justification of Reverse Discrimination“. Social Theory and Practice 3 (3), 289-306.

Gräfrath, Bernd. 1999. „Umgekehrte Diskriminierung“. In Halbe-Halbe? Zur Gerechtigkeit der Frauenquote, herausgegeben von Susanne Boshammer und Matthias Kayß, 50-74. Münster: LIT. 
Hartocollis, Anemona, und Stephanie Saul. 2017. „Affirmative Action Battle Has a New Focus: Asian-Americans“. In: New York Times, https://www.nytimes. com/2017/08/02/us/affirmative-action-battle-has-a-new-focus-asian-americans.html (zuletzt abgerufen am 26.5.2019).

Herrmann, Martina. 1999. „Quotierung als Mittel gerechter Verteilung“. In Halbe-Halbe? Zur Gerechtigkeit der Frauenquote, herausgegeben von Susanne Boshammer und Matthias Kayß, 75-98. Münster: LIT.

Hettinger, Edwin. 1987. „What is Wrong with Reverse Discrimination?“. Business and Professional Ethics Journal 6 (3), 39-55.

Hull, George. 2015 „Affirmative Action and the Choice of Amends“. Philosophia 43 (1), 113-134.

Hutchison, Katrina. 2013. „Sages and Cranks: The Difficulty of Identifying First-Rate Philosophers". In Women in Philosophy: What Needs to Change?, herausgegeben von Katrina Hutchison und Fiona Jenkins, 103-126. Oxford et al.: Oxford University Press.

Jenkins, Fiona. 2013. „Singing the Post-discrimination Blues: Notes for a Critique of Academic Meritocracy“. In Women in Philosophy: What Needs to Change?, herausgegeben von Katrina Hutchison und Fiona Jenkins, 81-102. Oxford et al.: Oxford University Press.

Klonschinski, Andrea. 2018. „\#Metoo und Frauen in der akademischen Philosophie: Der perfekte Sturm“. praefaktisch.de, https://www.praefaktisch.de/metoo/metoo-und-frauen-in-der-akademischen-philosophie-der-perfekte-sturm/ (zuletzt abgerufen am 26.5.2019).

Lai, Calvin et al. 2014. „Reducing implicit racial preferences: I. A comparative investigation of 17 interventions“. Journal of Experimental Psychology: General, 143, 1765-1785.

Leslie, Sarah-Jane, Andrei Cimpian, Meredith Meyer und Edward Freeland. 2015. „Expectations of Brilliance Underlie Gender Distributions Across Academic Disciplines“. Science 347, 6219, 262-265.

Leuschner, Anna. 2019. „Why So Low? On Indirect Effects of Gender Bias in Philosophy“. Metaphilosophy 50 (3), 231-249.

Leuschner, Anna. 2015. „Social exclusion in academia through biases in methodological quality evaluation: On the situation of women in science and philosophy“. Studies in History and Philosophy of Science 54, 56-63.

Lippert-Rasmussen, Kasper. 2014. Born Free and Equal? A Philosophical Inquiry into the Nature of Discrimination. Oxford et al.: Oxford University Press.

Mackenzie, Catriona, und Cynthia Townley. 2013. „Women In and Out of Philosophy“. In Women in Philosophy: What Needs to Change?, herausgegeben von Katrina Hutchison und Fiona Jenkins, 164-179. Oxford et al.: Oxford University Press.

Meshelski, Kristina. 2016. „Procedural Justice and Affirmative Action“. Ethical Theory and Moral Practice 19 (2), 425-443. 
Mikkola, Mari. 2013. „Die Society for Women in Philosophy (SWIP) Germany“. Newsletter Deutsche Gesellschaft für Philosophie 20, 4-6.

Nagel, Thomas. 1973. „Equal Treatment and Compensatory Discrimination“. Philosophy and Public Affairs, 2 (4), 348-363.

Newton, Lisa. 1973. „Reverse Discrimination as Unjustified“. Ethics 83 (4), 308-312.

Pojman, Louis. 1998. „The Case Against Affirmative Action“ International Journal of Applied Philosophy 12 (1), 97-115.

Rössler, Beate (Hg.). 1993. Quotierung und Gerechtigkeit. Frankfurt a.M./New York: Campus.

Rössler, Beate. 1999. „Quotierung als moralisches Problem“. In Halbe-Halbe? Zur Gerechtigkeit der Frauenquote, herausgegeben von Susanne Boshammer und Matthias Kayß, 33-49. Münster: LIT.

Saul, Jennifer. 2013. „Implicit Bias, Stereotype Threat, and Women in Philosophy“. In Women in Philosophy: What Needs to Change?, herausgegeben von Katrina Hutchison und Fiona Jenkins, 39-60. Oxford et al.: Oxford University Press.

Sesardic, Neven, und Rafael de Clerq. 2014. „Women in Philosophy: Problems with the Discrimination Hypothesis“. Academic Questions 27 (4), 461-473.

StZG: https://www.gesetze-im-internet.de/stzg/index.html

Thomson, Judith. 1973. „Preferential Hiring“. Philosophy and Public Affairs 2 (4), 364-384.

TierSchG: https://www.gesetze-im-internet.de/tierschg/BJNR012770972.html

WMA Declaration of Helsinki: https://www.wma.net/policies-post/wma-declaration-of-helsinki-ethical-principles-for-medical-research-involving-human-subjects/ 